\title{
EXISTENCE OF POSITIVE SOLUTION FOR A CLASS OF NONLOCAL PROBLEM WITH STRONG SINGULARITY AND LINEAR TERM
}

\author{
AI-JUN HOU AND JIA-FENG LIAO
}

Abstract. We consider a class of nonlocal problem with strong singularity and linear term. Combining with the variational method and Nehari manifold, a necessary and sufficient condition that shows the existence of positive solution is obtained.

Mathematics subject classification (2010): 35B09, 35D30, 35 J75.

Keywords and phrases: Nonlocal problem, strong singularity, linear term, variational method, Nehari manifold.

\section{REFERENCES}

[1] A. David And M.M. Lourdes, Multiplicity of solutions for a Dirichlet problem with a strongly singular nonlinearity, Nonlinear Anal., 95 (2014) 281-291.

[2] I. EKeland, On the variational principle, J. Math. Anal. Appl., 47(1974) 324-353.

[3] A. FisCELla, A fractional Kirchhoff problem involving a singular term and a critical nonlinearity, Adv. Nonlinear Anal., 1, 8(2019) 645-660.

[4] C.F. GUI AND F.H. Lin, Regularity of an elliptic problem with a singular nonlinearity, Proc. Roy. Soc. Edinburgh Sect. A., 6, 123(1993) 1021-1029.

[5] J. Hernández, F.J. MANCEbo And J.M. Vega, Positive solutions for singular nonlinear elliptic equations, Proc. Roy. Soc. Edinburgh Sect. A., 1, 137(2007) 41-62.

[6] N. Hirano, C. SACCON AND N. SHIOJI, Brezis-Nirenberg type theorems and multiplicity of positive solutions for a singular elliptic problem, J. Differential Equations, 3, 245(2008) 1997-2037.

[7] N. HIRANO, C. SACCON AND N. SHIOJI, Existence of multiple positive solutions for singular elliptic problems with concave and convex nonlinearities, Advances in Differential Equations, 3, 9(2004) 197-220.

[8] G. KirChHOFF, Mechanik, Teubner, Leipzig, 1883.

[9] A.C. LAZER AND P.J. MCKENNA, On a singular nonlinear elliptic Boundary-value problem, Proc. Amer. Math. Soc., 3, 111(1991) 721-730.

[10] C.Y. LEI, J.F. LiAO AND C.L. TANG, Multiple positive solutions for Kirchhoff type of problems with singularity and critical exponents, J. Math. Anal. Appl., 1, 421(2015) 521-538.

[11] C.Y. LEI AND J.F. LIAO, Multiple positive solutions for Kirchhoff type problems with singularity and asymptotically linear nonlinearities, Appl. Math. Lett., 94(2019) 279-285.

[12] C.Y. LeI AND J.F. LiaO, Multiple positive solutions for Schrödinger-Poisson system involving singularity and critical exponent, Math. Methods Appl. Sci., 7, 42(2019) 2417-2430.

[13] J.F. LiAO, X.F. KE, C.Y. LEI AND C.L. TANG, A uniqueness result for Kirchhoff type problems with singularity, Appl. Math. Lett., 59 (2016), 24-30.

[14] J.F. LIAO, P. ZHANG, J. LIU AND C.L. TANG, Existence and multiplicity of positive solutions for a class of Kirchhoff type problems with singularity, J. Math. Anal. Appl., 2, 430(2015) 1124-1148.

[15] J.F. LiaO, J. LiU, P. ZhANG AND C.L. TANG, Existence of two positive solutions for a class of semilinear elliptic equations with singularity and critical exponents, Ann. Polon. Math., 3, 116(2016) 273-292.

[16] J.L. LIONS, On some questions in boundary value problems of mathematical physics, Contemporary developments in continuum mechanics and partial differential equations (Proc. Internat. Sympos., Inst. Mat., Univ. Fed. Rio de Janeiro, Rio de Janeiro, 1977) pp. 284-346. 
[17] X. LIU AND Y.J. Sun, Multiple positive solutions for Kirchhoff type problems with singularity, Commun. Pure Appl. Anal., 2, 12(2013) 721-733.

[18] Y.J. Sun, Compatibility phenomena in singular problems, Proc. Roy. Soc. Edinburgh Sect. A., 6, 143(2013) 1321-1330.

[19] Y.J. SUN AND S.P. WU, An exact estimate result for a class of singular equations with critical exponents, J. Funct. Anal., 5, 260(2011) 1257-1284.

[20] Y.J. Sun, S.P. Wu AND Y.M. LonG, Combined effects of singular and superlinear nonlinearities in some singular boundary value problems, J. Differential Equations, 1, 176(2001) 511-531.

[21] Y.J. SUN AND D.Z. ZHANG, The role of the power 3 for elliptic equations with negative exponents, Calc. Var. Partial Differential Equations, 3-4, 49(2014) 909-922.

[22] Y.J. Sun AND Y.X. TAN, Kirchhoff type equations with strong singularities, Commun. Pure Appl. Anal., 1, 18(2019) 181-193.

[23] D.C. WANG AND B.Q. YAN, A uniqueness result for some Kirchhoff-type equations with negative exponents, Appl. Math. Lett., 92(2019) 93-98.

[24] X. WANG, X.Q. QIN AND G. HU, Existence of weak positive solution for a singular elliptic problem with supercritical nonlinearity, Anal. Math. Phys. 1, 8(2018) 43-55.

[25] X. WANG, L. ZHAO AND P.H. ZHAO, Combined effects of singular and critical nonlinearities in elliptic problems, Nonlinear Anal., 87(2013) 1-10.

[26] H.T. YANG, Multiplicity and asymptotic behavior of positive solutions for a singular semilinear elliptic problem, J. Differential Equations, 2, 189(2003) 487-512.

[27] Q. ZHANG, Existence of positive solution to Kirchhoff-Schrödinger-Poisson system with strong singular term, J. Math. Phys., 60, 041504(2019) 1-11. 